\title{
Magnetothermoelectric properties of the degenerate semiconductor HgSe:Fe
}

\author{
B. Tieke \\ Research Institute for Materials, High Field Magnet Laboratory, University of Nijmegen, 6500 GL Nijmegen, The Netherlands \\ R. Fletcher \\ Physics Department, Queen's University, Kingston, Ontario, Canada, K7L 3N6 \\ J. C. Maan \\ Research Institute for Materials, High Field Magnet Laboratory, University of Nijmegen, 6500 GL Nijmegen, The Netherlands \\ W. Dobrowolski, A. Mycielski, and A. Wittlin \\ Institute of Physics, Polish Academy of Science, Aleja Lotników 32/46, 02-668 Warsaw, Poland
}

(Received 3 April 1996)

\begin{abstract}
There are few previous data on the magnetothermoelectric properties of degenerate semiconductors. Although magnetic quantum oscillations in the thermopower have been previously studied, quantitative, or even semiquantitative agreement with theory was not achieved. We have developed a theoretical model allowing a quantitative description of both the oscillatory and nonoscillatory parts of the thermopower tensor $S$, and have studied its validity in an experimental system. For the experiments we have chosen a high mobility sample of HgSe:Fe $\left(n=4.6 \times 10^{24} \mathrm{~m}^{-3}\right)$, and have measured both of the independent components of $S$ in a transverse magnetic field $B$ of $\leqslant 8 \mathrm{~T}$ at temperatures $1 \mathrm{~K}<T<60 \mathrm{~K}$, together with the longitudinal and Hall resistivities and the thermal conductivity. Our model predicts that the transverse component $S_{y x}$ is not affected by phonon drag, and all our data are in agreement with this. The absolute value of the nonoscillating part of $S_{y x}$ depends linearly on $T$ consistent with domination by diffusion. We observe large oscillations in $S_{y x}$ which exhibit a phase shift of $\pi / 2$ relative to the resistivity oscillations. By including elastic electron scattering in the diffusion theory we obtain excellent quantitative agreement for both the oscillations and the smooth background under all conditions of $B$ and $T$, including the observed phase shift of $\pi / 2$. In contrast to $S_{y x}$, the longitudinal component $S_{x x}$ shows a large contribution from phonon drag over most of the temperature range. $S_{x x}$ exhibits quantum oscillations similar to $S_{y x}$, but these are found to be in phase with those in the resistivity and are far too large to be explained by diffusion in any available model. Instead, they are well described by oscillations in electron-phonon scattering which modulates phonon drag in much the same way as is observed in twodimensional systems. Small oscillations are also observed in the lattice thermal conductivity and their magnitude is shown to be consistent with the same phonon-electron scattering being responsible. Our analysis demonstrates that the study of the thermopower is a powerful tool in the understanding of both the elastic (through $S_{y x}$ ) as well as the inelastic (through $S_{x x}$ ) scattering of electrons in degenerate semiconductors. [S0163-1829(96)03440-6]
\end{abstract}

\section{INTRODUCTION}

A full understanding of the magnetothermopower of degenerate semiconductors has been slower to emerge than for the case of the resistivity. The lack of progress is partly because thermoelectricity is a more subtle phenomenon, but also because the experiments are more difficult to carry out. As far as we are aware, there is no comprehensive set of data available on either the oscillatory or nonoscillatory parts for both of the independent components of the thermopower of a degenerate semiconductor in a transverse magnetic field. This paper presents such a set using $\mathrm{HgSe}$ doped with $\mathrm{Fe}$, chosen because it has a high mobility and is known to give large oscillations in the resistivity.

The early literature on thermomagnetic effects in degenerate semiconductors is summarized in reviews by Zyryanov and Guseva ${ }^{1}$ and Puri and Geballe, ${ }^{2}$ the latter dealing mainly with very low carrier density systems which can be taken into the high-field limit, i.e., only the last Landau level is occupied. The present work is concerned with the regime where many levels are occupied. A significant complication with thermopower is that it is much more sensitive to phonon drag than is resistivity, and it is often not clear what fraction of the experimental thermopower, especially in the case of oscillations, is to be ascribed to each of the two components, phonon drag and diffusion. As far as we are aware, all this early work assumed that the oscillatory phenomena were due only to diffusion, and achieved limited quantitative success.

More recent work turned to normal metals and twodimensional electron gases (2DEG's). The latter are usually degenerate metals under the conditions of measurement for oscillatory phenomena (a recent review ${ }^{3}$ gives many details) and have much in common with bulk degenerate semiconductors. The main difference is that, with a 2DEG, 3D phonons interact with $2 \mathrm{D}$ electrons, but in semiconductors both systems are interpenetrating and 3D. In each case the 
phonon system is only weakly affected by electronic scattering. With 2DEG's it rapidly became clear that, under normal conditions, phonon drag is the dominant cause of the oscillations in the longitudinal thermopower (which we will refer to simply as the thermopower). Diffusion oscillations have never been reliably seen in the thermopower, though they have been observed in the transverse thermopower ${ }^{4}$ (usually called the Nernst-Ettingshausen or NE coefficient).

Experiments on metals ${ }^{5-7}$ showed that phonon drag plays little part in thermoelectric oscillations. Excellent agreement between theory and experiment was achieved for both the thermopower and NE coefficient by considering only diffusion effects. With suitable modifications, the work on metals provides the theoretical basis for some of the present analysis.

The fact that the oscillatory thermopower is dominated by phonon drag in 2DEG's but by diffusion in metals is easily understood. Phonon drag arises via electron-phonon coupling, and so is expected to oscillate with roughly the same relative magnitude as the electronic density of states at the Fermi level, at least to first order. In normal metals such oscillations are at the level of $1 \%$, but in 2DEG's they vary up to $100 \%$. Large density-of-state oscillations are also present in degenerate semiconductors, so one might expect these to be reflected in phonon drag oscillations in this case too.

The question then arises of how one makes a separation between phonon drag and diffusion oscillations with a degenerate semiconductor. The surprising answer is that the system itself makes such separation. Our model calculation shows that the NE coefficient is not influenced by phonon drag, and we will demonstrate experimentally that this is indeed true within experimental error. This remarkable result enables us to examine the diffusion thermopower in some detail, and we find that calculations based solely on diffusion reproduce the experimental data almost perfectly for both the oscillatory and nonoscillatory parts.

In contrast, $S_{x x}$ is dominated by phonon drag, including the oscillations, at least at relatively low temperatures. Previous work ${ }^{8}$ on $\mathrm{HgSe}$ followed the accepted wisdom, and analyzed these oscillations in terms of diffusion, but our data show that this approach is inappropriate. We are able to give a semiquantitative explanation of the oscillatory part of $S_{x x}$ at high fields and low temperatures in terms of phonon drag.

\section{THEORY}

We will be interested in the components of the transport tensors $\sigma, \epsilon, \Pi$, and $\lambda$ (for which the field is perpendicular to the current) as defined by the relations

$$
\begin{aligned}
& \vec{J}=\sigma \vec{E}-\epsilon \nabla T, \\
& \vec{U}=\Pi \vec{E}-\lambda \nabla T,
\end{aligned}
$$

where $\vec{J}$ and $\vec{U}$ are the electric and heat current densities due to an applied electric field $\vec{E}$ (more generally, $\vec{E}$ is the negative of the gradient of the electrochemical potential) and temperature gradient $\nabla T$. The thermopower tensor $S$ is defined according to $\vec{E}=S \nabla T$, so that, from Eq. (1) with the boundary condition $\vec{J}=0, S=\sigma^{-1} \epsilon=\rho \epsilon$, where $\rho$ is the resistivity tensor. The magnetic field is taken to be along $z$, and it is assumed throughout that there is sufficient symmetry so that $\sigma_{x x}=\sigma_{y y}, \sigma_{y x}=-\sigma_{x y}$, etc. In the present case the heat conduction is primarily by phonons, and we initially assume $\nabla T_{y}=0$ (later we will make a correction for this not holding exactly). This is the same condition as for 2DEG's where the insulating substrate carries the heat. Therefore, the experiments measure the components of $S_{i j}$ directly. Thus the thermopower is just $S_{x x}$ and the NE coefficient is $S_{y x}$ as follows:

$$
\begin{aligned}
& S_{x x}=E_{x} / \nabla T_{x}=\rho_{x x} \epsilon_{x x}+\rho_{y x} \epsilon_{x y}, \\
& S_{y x}=E_{y} / \nabla T_{x}=\rho_{x x} \epsilon_{y x}+\rho_{y x} \epsilon_{x x} .
\end{aligned}
$$

In bulk metals the heat current is mainly carried by electrons, which means that there is a transverse temperature gradient $\nabla T_{y}$ due to the Lorentz, force and so the above equations are not valid in this simple form.

The thermoelectric tensor $\epsilon$ has two contributions, diffusion $\epsilon^{d}$ and phonon drag $\epsilon^{g}$, which are linearly additive; this results in their contributions to $S$ also being additive, i.e. $S=S^{d}+S^{g}$. Throughout, oscillatory parts (i.e., the part resulting from the effects of magnetic quantization of the electrons) are denoted by a tilde, e.g. $\widetilde{\epsilon}$, and nonoscillatory parts by a bar, e.g. $\bar{\epsilon}$.

The diffusion component is examined first under the assumption that elastic scattering is dominant in $\sigma$. Because the electron gas is degenerate, $\bar{\epsilon}^{d}$ can be calculated from the Mott relation ${ }^{9}$ relation $\bar{\epsilon}^{l}=-L_{o} e T(\partial \bar{\sigma} / \partial \varepsilon)_{\varepsilon_{F}}$ and with spherical energy-band results for the conductivity tensor $\bar{\sigma}$ one finds

$$
\begin{gathered}
\bar{\epsilon}_{x x}^{d}=\sigma_{x x} \frac{L_{o} e T}{\varepsilon_{F}}\left[\frac{3}{2}+p \frac{1-\beta^{2}}{1+\beta^{2}}\right], \\
\bar{\epsilon}_{y x}^{d}=\sigma_{y x} \frac{L_{o} e T}{\varepsilon_{F}}\left[1+\frac{2 p}{1+\beta^{2}}\right],
\end{gathered}
$$

where $p=(\partial \ln \tau / \partial \ln \varepsilon)_{\varepsilon_{F}} ; e$ is the electron charge taken to be negative, i.e., $e=-|e| ; \varepsilon_{F}$ is the Fermi energy; $\beta=\omega_{c} \tau$, with $\tau$ the transport lifetime and $\omega_{c}$ the cyclotron frequency; $L_{o}$ is the Lorenz number $\pi^{2} k_{B}^{2} / 3 e^{2}$, and $k_{B}$ is the Boltzmann constant.

With Eqs. (2) one finds the following expressions for $\bar{S}_{x x}^{d}$ and $\bar{S}_{y x}^{d}$ as a function of magnetic field:

$$
\begin{gathered}
\bar{S}_{x x}^{d}=\frac{L_{o} e T}{\varepsilon_{F}}\left[\frac{3}{2}+\frac{p}{1+\beta^{2}}\right], \\
\bar{S}_{y x}^{d}=\frac{L_{o} e T}{\varepsilon_{F}}\left[\frac{p \beta}{1+\beta^{2}}\right] .
\end{gathered}
$$

These field dependencies are essentially the same as with a $2 \mathrm{DEG},{ }^{10}$ with the former tending to the entropy per unit charge and the latter to zero as $B \rightarrow \infty$. Equivalent equations have been given by Kuleev et al. ${ }^{11}$

The oscillating part $\widetilde{\epsilon}^{d}$ will be obtained in terms of $\widetilde{\sigma}$ using a semiclassical approach introduced by Young, ${ }^{5}$ valid for elastic scattering. The derivation is analogous to that of the Mott result for the nonoscillatory parts, and in fact reduces to this when $k_{B} T \ll \hbar \omega_{c}$. One finds 


$$
\widetilde{\epsilon}^{d}=-i \frac{\pi k_{B}}{e} \frac{D^{\prime}(X)}{D(X)} \widetilde{\sigma}=\alpha \widetilde{\sigma},
$$

where $X=2 \pi^{2} k_{B}^{2} T / \hbar \omega_{c}, D(X)=X / \sinh X$ is the thermal damping factor for oscillations in $\sigma, D^{\prime}(X)=d D(X) / d X$ is the thermal damping factor for oscillations in $\epsilon$, and $i=\sqrt{-1}$ indicates a $\pi / 2$ difference in the phase of the oscillations. This phase difference is due to the fact that $\epsilon$ is essentially the derivative of $\sigma$ with respect to electronic energy, though thermal averaging must be included to make the relation precise. The calculation of the oscillating diffusion contribution $\widetilde{S}^{d}$ is similar to the case of the $2 \mathrm{DEG},{ }^{4}$ and the components are found to be

$$
\begin{gathered}
\widetilde{S}_{x x}^{d} \simeq \alpha\left(\tilde{\rho}_{x x} \bar{\sigma}_{x x}+\widetilde{\rho}_{y x} \bar{\sigma}_{y x}\right)=\frac{\alpha}{1+\beta^{2}}\left(\frac{\tilde{\rho}_{x x}}{\bar{\rho}_{x x}}+\beta^{2} \frac{\tilde{\rho}_{y x}}{\overline{\rho_{y x}}}\right), \\
\widetilde{S}_{y x}^{d} \simeq \alpha\left(\widetilde{\rho}_{x x} \bar{\sigma}_{y x}+\widetilde{\rho}_{y x} \bar{\sigma}_{x x}\right)=\frac{\alpha \beta}{1+\beta^{2}}\left(\frac{\tilde{\rho}_{x x}}{\overline{\rho_{x x}}}+\frac{\tilde{\rho}_{y x}}{\overline{\rho_{y x}}}\right),
\end{gathered}
$$

where $\bar{\rho}$ and $\tilde{\rho}$ are the nonoscillatory and oscillatory resistivities, and the last step again assumes spherical energy bands. If absolute results are required, the expressions for $\tilde{\rho}_{x x}$ and $\tilde{\rho}_{y x}$ from standard theories ${ }^{12}$ may be substituted. However, for our purposes the above form is the most convenient. The sums of Eqs. (4) and (6) give full results for the diffusion components.

Previous experimental results on degenerate semiconductors have been interpreted in terms of a different result for $\widetilde{S}_{x x}^{d}$ given by Obraztsov, ${ }^{13}$ and so we briefly describe this in the following. Obraztsov finds $S_{x x}^{d}=\mathcal{S} / n e$, where $\mathcal{S}$ is the entropy of the electron gas. When the oscillations are sinusoidal so that harmonics can be ignored, the ensuing result for $\widetilde{S}_{x x}^{d}$ can be written in terms of the relative amplitude of the oscillations in the density of states at the Fermi level $\widetilde{\nu} / \bar{\nu}$ (which we obtain from Ref. 12) as

$$
\frac{\widetilde{S}_{x x}^{d}}{\overline{S_{x x}^{d}(\infty)}}=-\frac{3}{X} D^{\prime}(X) \frac{\tilde{\nu}}{\bar{\nu}},
$$

where $\bar{S}_{x x}^{d}(\infty)$ is the high field limit of Eq. (4a), i.e., $\bar{S}_{x x}^{d}(\infty)=\pi^{2} k_{B}^{2} T / 2 e \varepsilon_{F}$. No result is given for $\widetilde{S}_{y x}^{d}$. Obraztsov made his calculation in the zero-scattering limit and thus there was no impurity damping term. Schroder and Landwehr ${ }^{8}$ added such a term, since it appears to be essential from a physical point of view. By writing the result as above, the damping term is automatically included in $\widetilde{\nu} / \bar{\nu}$. There is a very useful limit of this equation. As $X \rightarrow 0$, then $D^{\prime}(X) \rightarrow-X / 3$. In this case we obtain the simple result $\widetilde{S}_{x x}^{d} / \bar{S}_{x x}^{d}(\infty)=\widetilde{\nu} / \bar{\nu}$. This is a good approximation when $X<1$. For larger $X$ the ratio $\widetilde{S}_{x x}^{d} / \bar{S}_{x x}^{d}(\infty)$ is always smaller, and for large $X, D^{\prime}(X) \rightarrow-2 X \exp (-X)$. We note that the predicted phase shift is always $\pi$ compared to $\widetilde{\rho}_{x x}$. One might assume that Eqs. (7) and (6) are complementary and perhaps valid under different conditions, but as far as we are aware this is not the case (the Appendix has further details).

We do not have as detailed a theory for the phonon drag part $S^{g}$. However, Zaremba ${ }^{14}$ showed that it is possible to write the phonon drag components of the thermoelectric ten- sor $\epsilon^{g}$ in the form $\epsilon^{g}=\sigma C$, where $C$ is a constant which contains all the details of the electron-phonon scattering, and $\sigma$ is the conductivity tensor exactly as used above. The theory includes the classical effect of the Lorentz force through $\sigma$, but does not take into account magnetic quantization, so $C$ is not a function of $B$. From this definition it follows that $C=S_{o}^{g}$ i.e., the constant is just the phonon drag thermopower at zero field. Because of magnetic quantization, we expect the phonon-electron scattering probability to oscillate, and we assume this can be allowed for by writing $C=\bar{C}+\widetilde{C}$. When $k_{B} T \ll \hbar \omega_{c}$, it is reasonable to assume that $\widetilde{C} / \bar{C}=\gamma \widetilde{\nu} / \bar{\nu}$, where $\gamma$ is a constant of order unity. This is analogous to the case of elastic scattering where a similar result holds. At higher temperatures $\gamma$ will contain a thermal damping term analogous to $D^{\prime}(X)$, but it will presumably be a different function for phonon drag, which is an inelastic interaction. Thus using Eq. (2) we find

$$
\begin{gathered}
S_{x x}^{g}=S_{o}^{g}(1+\gamma \widetilde{\nu} / \widetilde{\nu}), \\
S_{y x}^{g}=0 .
\end{gathered}
$$

In the case of $S_{y x}^{g}$ the two terms in Eq. (2b) exactly cancel, independent of field. Thus $S_{y x}^{g}$ is predicted to have no phonon drag contribution for either the background or the oscillatory part. The physical reason for this result is as follows. The fact that $\epsilon^{g}=\sigma C$ means that the electric current produced by phonon drag in Eq. (1a) is equivalent to that from a fictitious electric field parallel to $\nabla T$ (assuming electrons). Thermopower is measured with no resulting electric current so a real electric field is set up $[\vec{E}$ of Eq. (1a)] to provide the compensating current. Clearly this measured field is equal and opposite to the fictitious one, i.e., antiparallel to $\nabla T$, which has a component only along $x$ in the present case.

The results also apply to the case of a 2DEG, and the nonoscillatory parts are essentially the same as those predicted by Zianni, Butcher, and Kearney, ${ }^{15}$ but it is known experimentally ${ }^{10}$ that $\bar{S}_{y x}^{g} \neq 0$ in this case, though it is small compared to either of the terms in Eq. (2b). In bulk metals $\nabla T_{y} \neq 0$, and so the measured NE coefficient is a mixture of $S_{x x}$ and $S_{y x}$, and the phonon drag part is not expected to be zero.

\section{EXPERIMENTS}

Data were obtained for two samples, but we report on only the one for which we have a reasonably complete set of results on all the relevant coefficients. The other sample gave consistent data for the coefficients measured. The HgSe:Fe crystals were grown by the Bridgeman method at the Polish Academy of Science. The sample was oriented using standard Laue techniques and cut with a wire-erosion machine into the shape of a rectangular parallelepiped, with dimensions $9.0 \times 2.7 \times 0.80 \mathrm{~mm}^{3}$. The electron density was found to be $4.6 \times 10^{24} \mathrm{~m}^{-3}$ independent of temperature, and the mobility $4.0 \mathrm{~m}^{2} / \mathrm{V} \mathrm{s}$ at $4 \mathrm{~K}$, decreasing with increasing temperature to $\sim 3.2 \mathrm{~m}^{2} / \mathrm{V} \mathrm{s}$ at $50 \mathrm{~K}$. Using an effective mass $^{16,17}$ of $m^{*}=0.065 m_{e}$ gives a Fermi energy $\varepsilon_{F} \sim 0.155$ $\mathrm{eV}$ or $1800 \mathrm{~K}$, so the gas is highly degenerate at all temperatures in these experiments. The [100] direction was perpendicular to the large face, and this was also the direction of the 
magnetic field $B$. A brief immersion in a solution of $5 \% \mathrm{Br}$ in methanol produced polished surfaces.

The sample was indium soldered into the cryostat, and a small strain gauge was epoxied to the free end for use as a heater. Ag filled epoxy was used to make contacts to four 50 $\mu$ manganin wires for use as potential leads; two similar wires were soldered with indium to act as current leads for resistivity measurements. The temperature and temperature gradient were measured with two $22-\mathrm{k} \Omega$ Philips surface mount resistors epoxied to the sample. These are useful from about $1-100 \mathrm{~K}$, and have negligible magnetoresistance over the whole temperature and field range $(B \leqslant 8 \mathrm{~T})$. Calibrations were made using a commercial Ge resistance thermometer. Temperature was controlled with a capacitance thermometer which is insensitive to magnetic field.

An EM dc nanovoltmeter was used to measure potential differences across the sample. In all measurements in a magnetic field, suitable averages were evaluated for data taken at $\pm B$ to eliminate unwanted components, though these components were typically small.

The thermal conductivity of the samples was field dependent over the whole range of temperatures. This was typically a 5-30\% effect, and led to changes of the temperature gradient and the average temperature of the sample during field sweeps. Complete data on both of these effects were obtained, and the thermopower was corrected for the change in gradient (on a continuous basis as a function of $B$ ), but not for the change in absolute temperature. This latter is negligible above about $15 \mathrm{~K}$, and peaks at a few tenths of a degree near $6 \mathrm{~K}$

The main uncertainties in the data are systematic and arise from the finite size of the potential and thermometer contacts. The latter contributes an uncertainty of $\sim 10 \%$ in the temperature gradient. The former gives a $\sim 10 \%$ uncertainty in the longitudinal electric field, but perhaps only a very small error in the transverse field. Thus we expect uncertainties of $\sim 10 \%$ in any transverse thermal quantity (but probably much less for the Hall resistivity), $\sim 10 \%$ for the thermal conductivity and longitudinal resistivity, and $\sim 15 \%$ for the thermopower. Errors due to thermometer calibrations should be small over most of the range, but might become serious at the highest temperatures because of the poor sensitivity of the Philips resistors and the calibrating thermometer. At the upper limit $\sim 100 \mathrm{~K}$, this might lead to further errors of $\sim 10 \%$ in the thermal quantities. All other errors are believed to be small in comparison.

\section{RESULTS AND DISCUSSION}

Field sweep data for most of the transport coefficients were taken over the range $1-55 \mathrm{~K}$, but thermopower and thermal conductivity were measured up to about $100 \mathrm{~K}$ in zero field. This paper is primarily concerned with thermoelectric effects, but other coefficients are also important to the analysis. We first briefly review these auxiliary measurements.

\section{A. Auxiliary measurements}

Figure 1 gives some representative data on $\rho_{x x}$ as a function of field at various temperatures. We are mainly inter-

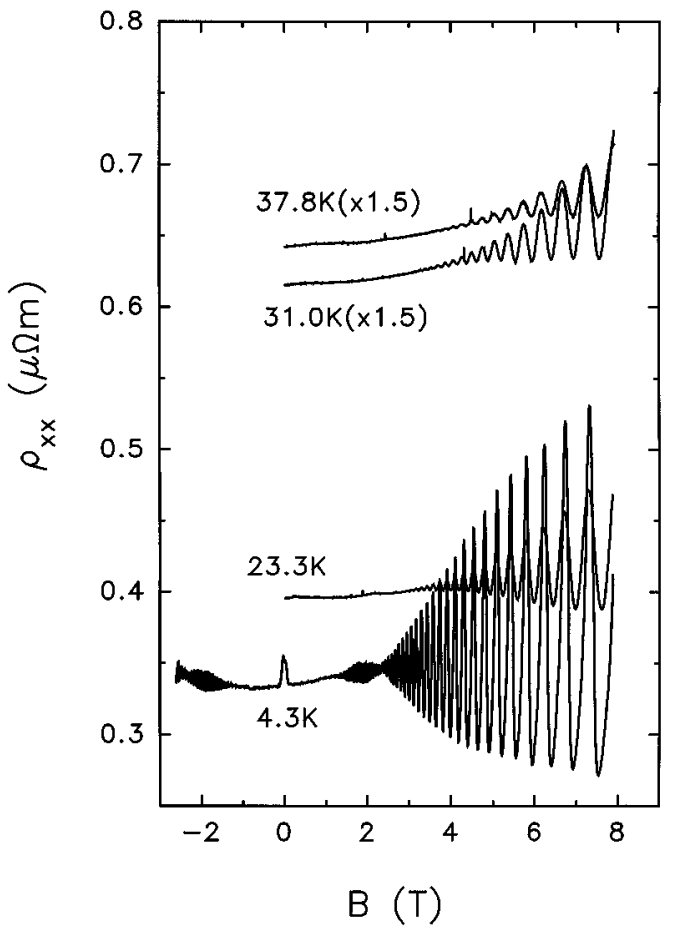

FIG. 1. A selection of $\rho_{x x}$ data at various temperatures. The upper two curves have been multiplied by 1.5 for clarity.

ested in such data for comparison with $\widetilde{S}$ later. The modulation in the amplitude of the oscillations is known to arise from the lack of inversion symmetry of the zinc-blende crystal structure (see Ref. 17 for recent data and analysis), but the details are not relevant to the present work. A useful quantity for later use is the relative modulation in the electronic density of states $\widetilde{\nu} / \bar{\nu}$ at the Fermi level due to magnetic quantization. Using the results of Roth and Argyres, ${ }^{12}$ who find $\tilde{\rho}_{x x} / \bar{\rho}_{x x}=(5 / 2) D(X) \widetilde{\nu} / \bar{\nu}$ and the data in Fig. 1, we estimate the peak-peak value of $\widetilde{\nu} / \bar{\nu}$ to be $\sim 0.4$ at $8 \mathrm{~T}$.

The Hall resistivity $\bar{\rho}_{y x}$ is linear in $B$ over the range $1-50$ $\mathrm{K}$, and shows that any variation in carrier concentration with temperature is no more than $1 \%$. Small oscillations were superposed on the smooth background. The oscillatory component $\widetilde{\rho}_{y x}$ is expected to be very small when $\beta \gg 1$. At low temperatures our experiments give a peak-peak value of $\tilde{\rho}_{y x} / \bar{\rho}_{y x} \sim-5 \times 10^{-3}$ at $8 \mathrm{~T}$.

Ignoring a negligible thermoelectric correction (arising from the fact that thermal conductivity is measured with $\vec{J}=0$ rather than $\vec{E}=0$ ), the measured thermal conductivity is just $\lambda$ that appears in Eq. (1). We denote the zero-field value of $\lambda_{x x}$ as $\lambda_{o}$ and Fig. 2 shows this as a function of temperature. The data are very similar to those obtained by Whitsett $e t a l .{ }^{18}$ for $\mathrm{HgSe}$ samples which did not contain Fe. The conductivity is dominated by phonons, say $\lambda_{o}^{g}$ at low temperatures, but by about $20 \mathrm{~K}$ the electronic contribution $\lambda_{o}^{e}$ becomes visible (see below). The solid line is a calculation of $\lambda_{o}^{g}$ using the same model and phonon-scattering mechanisms as Whitsett et al., which include boundaries, electrons, Rayleigh, resonant scattering by quasilocalized phonons, and $N$ and $U$ phonon-phonon processes. We list the fitting parameters, since they should be useful in any future calculation of the phonon drag thermopower of this sample, 


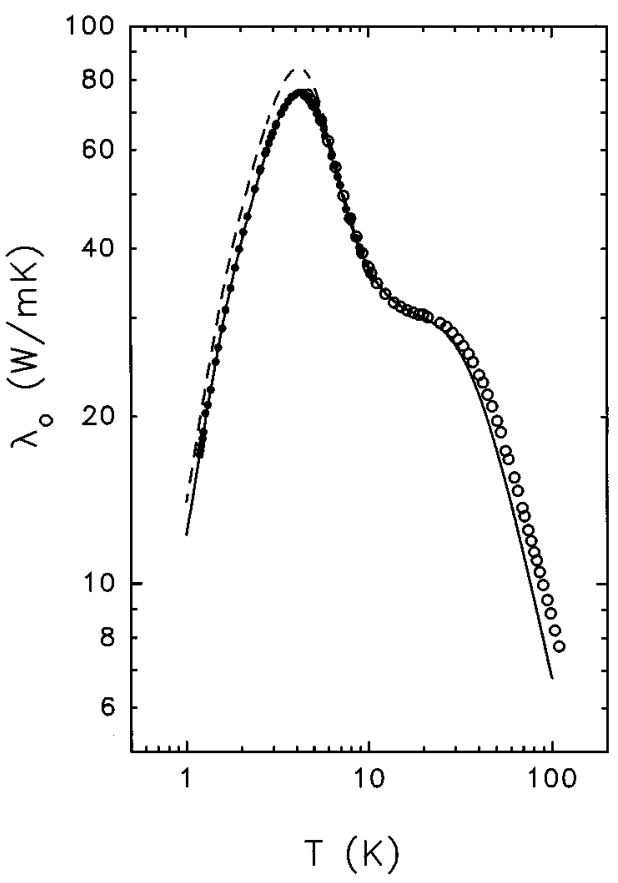

FIG. 2. The measured zero-field thermal conductivity $\lambda_{o}$ as a function of temperature. The two sets of symbols correspond to two different cooldowns. The solid line is fitted using the model of Whitsett et al., as described in the text, for the lattice thermal conductivity $\lambda_{o}^{g}$. The dashed line shows the effect of turning off the phonon-electron scattering in the calculation.

but we refer the reader to the paper by Whitsett $e t$ al. for full details and definitions: boundaries $L=1.50 \mathrm{~mm}$ (after applying the correction of Wybourne, Edison, and Kelly, ${ }^{19}$ ) and a specularity factor $F=1.4$ : Rayleigh $A=2.9 \times 10^{-42} \mathrm{~s}^{3}$ : resonance $\omega_{s}=5.1 \times 10^{12} \mathrm{~s}^{-1}, \quad \omega_{\alpha}=3.3 \times 10^{12} \mathrm{~s}^{-1}, \quad$ and $N=7.5 \times 10^{27} \mathrm{~m}^{-3}: U$ process $B_{1}^{\prime}=1.7 \times 10^{-17} \mathrm{~s} / \mathrm{K}$. Our $N$ process and electronic scattering are identical to those in Whitsett et al., with the latter restricted to the range of phonon wave vectors less than the diameter of the Fermi circle. Our values for boundary, Rayleigh, and $U$-process scattering are all very similar, but the strength of the resonance scattering $(N)$ is roughly a factor 2 higher. This last is responsible for the strong dip in $\lambda_{o}$ near $15 \mathrm{~K}$.

Figure 3 shows a selection of data on the thermal conductivity $\lambda_{x x}$, normalized to $\lambda_{o}$, as a function of field at various temperatures. The behavior of the nonoscillatory background is seen to be significantly different at low and high temperatures. At high temperatures there is a rapid drop in $\lambda_{x x}$ at low fields, followed by an approximate saturation. This is due to the electronic contribution $\lambda_{x x}^{e}$ Taking the electronic thermal conductivity tensor $\lambda^{e}=L T \sigma$, where $L$ is an effective Lorenz number, then, with $\nabla T_{y}=0$ (which is an approximation if $\lambda_{x x}^{e}$ is finite), one has $\lambda_{x x}=\lambda^{g}+L T \sigma_{o} /\left(1+\beta^{2}\right)$. Thus the electronic contribution is suppressed by the Lorentz motion, which enables us to estimate its magnitude. It is visible only above $\sim 20 \mathrm{~K}$, and reaches about $10 \%$ of the total at $\sim 50 \mathrm{~K}$. Whitsett $e t$ al. ${ }^{18}$ quote similar results obtained by a different method.

The difference in curve shapes in Fig. 3 between 4 and 15 $\mathrm{K}$ implies that the decrease in $\lambda_{x x}$ is due to a different mechanism. Above $20 \mathrm{~K}$ the saturation field is rather low,

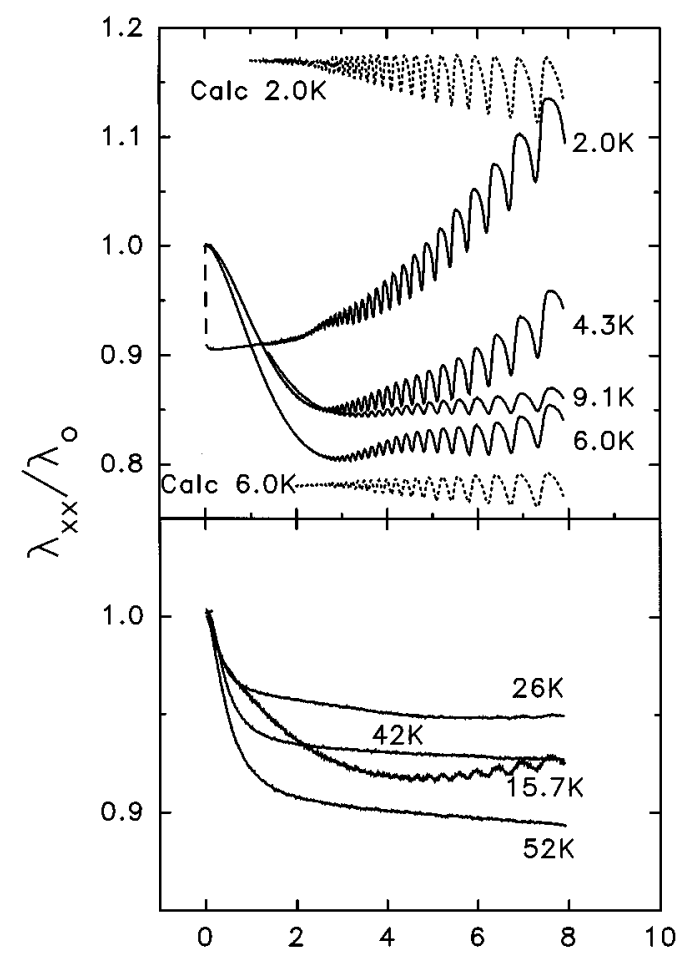

FIG. 3. The thermal conductivity $\lambda_{x x}$ as a function of field at various temperatures. The data are normalized to the zero-field values $\lambda_{o}$. In the upper panel the dotted curves are calculations of the oscillations at 2 and $6 \mathrm{~K}$ assuming they are due to $p$-e scattering.

and is governed by $\omega_{c} \tau=1$; this field slowly increases with temperature due to the temperature dependence of $\mu$. Below $20 \mathrm{~K}$, where the electronic contribution becomes negligible, another mechanism appears with a higher saturation field. This behavior may be related to the Fe impurities, though they do not seem to be connected with the dip in $\lambda_{o}$ at about $15 \mathrm{~K}$ seen in Fig. 2, since a similar dip is observed in undoped $\mathrm{HgSe} .{ }^{18}$ At even lower temperatures below $4.3 \mathrm{~K}$ the behavior is again different, with an abrupt drop in $\lambda$ at very low fields $(\sim 0.1 \mathrm{~T}$, as Fig. 3 shows $)$ which seems reminiscent of a phase change. The sharp decrease in $\lambda_{x x}$ is mirrored by a similar drop in $\rho_{x x}$ at the lowest temperatures.

The observed oscillations do not arise from oscillations in $\lambda^{e}$, which is negligible at low temperatures even before being turned off by the field, but are caused by the effect of magnetic quantization of the electrons on phonon-electron $(p-e)$ scattering. They are clearly related to the quantity $\gamma \widetilde{\nu} / \bar{\nu}$ that appears in Eq. (8a), and in Sec. IV B we will give an analysis based on this idea.

\section{B. Thermoelectric coefficients}

The thermopower $S_{x x}$ at zero field, $S_{o}$, is shown in Fig. 4. There is a small shift in the data between cooldowns which is not reflected in the thermal conductivity in Fig. 2. The change might be intrinsic to the electronic properties, but might also be simply due to instabilities in the potential leads because of the use of Ag-filled epoxy for the connections.

The straight line is the estimated diffusion part (explained below), and it is clear that phonon drag is completely dominant up to about $25 \mathrm{~K}$. An interesting feature is that the ratio 


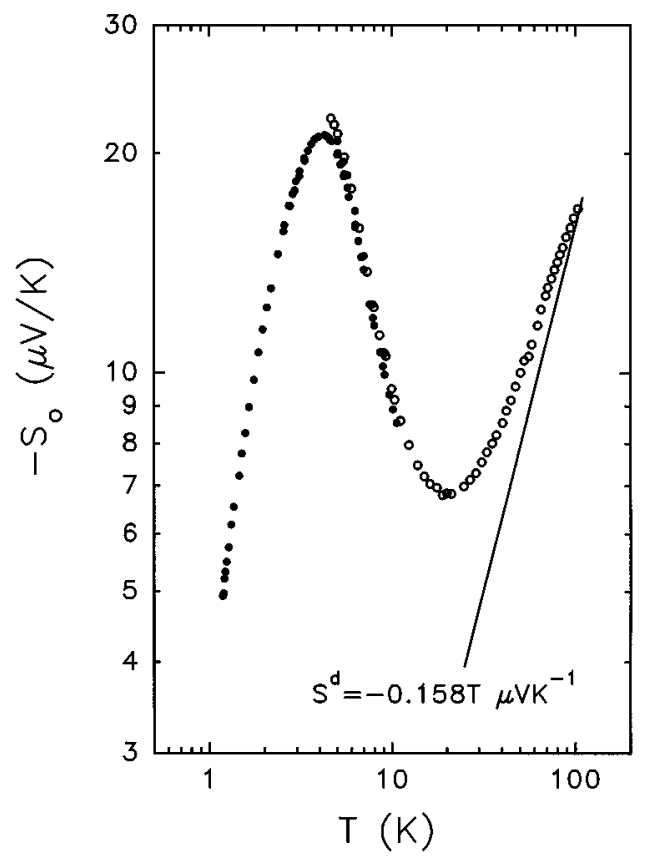

FIG. 4. The thermopower at zero field $S_{o}$ as a function of temperature. The two sets of symbols are the same two cooldowns as mentioned in Fig. 1. The straight line is the estimated diffusion component.

of $S_{o}$ and $\lambda$ is constant to a few percent over the range 1-6 $\mathrm{K}$. This is unlikely to be coincidental, and is probably a reflection of the fact that the same phonons are responsible for both $S_{o}$ and $\lambda_{o}$ in this temperature range. At higher temperatures most of the phonons can no longer interact with electrons. The cutoff phonon frequency for $p$-e scattering is related to the diameter of the Fermi sphere, and corresponds to $\sim 15 \mathrm{~K}$. The decrease in the ratio $S_{o} / \lambda$ above $\sim 6 \mathrm{~K}$ is presumably related to this.

Figure 5 gives a selection of data on $S_{y x}$ as a function of magnetic field at different temperatures. Kuleev et al. ${ }^{11}$ recently published data on $\bar{S}_{y x}$ for a series of $\mathrm{HgSe}: \mathrm{Fe}$ samples, but field dependencies were not given. According to Eq. (8) there should be no phonon drag in this coefficient, and the data do have the characteristics expected of the diffusion parts given in Eqs. (4b) for $\bar{S}_{y x}$ and $(6 \mathrm{~b})$ for $\widetilde{S}_{y x}$ as we will now show.

The nonoscillatory part of the experimental data have been fitted to Eq. (4b) to find the amplitude $L_{o} e T p / \varepsilon_{F}$ and the mobility $\mu=\beta / B$ as a function of temperature. These fits to the smooth parts are essentially perfect, even when the oscillation amplitude is large. We find $\mu$ to be consistently lower than calculated from the Hall and longitudinal resistivity, and ranges from $\sim 3.2 \mathrm{~m}^{2} / \mathrm{V} \mathrm{s}$ at low temperature to $\sim 1.7 \mathrm{~m}^{2} / \mathrm{V}$ s by $50 \mathrm{~K}$. The difference may be related to the distinction between the electronic relaxation times appropriate to momentum and thermal transport, which our theory does not distinguish. Figure 6 gives $L_{o} e T p / \varepsilon_{F}$ as a function of $T$. The observed linear relationship shows that diffusion is dominant and $p$ can be taken as constant. It should be noted that each term in the phonon drag part of Eq. (2b) has a magnitude of $S_{o}^{g} \beta /\left(1+\beta^{2}\right)$ which is of the same form as the diffusion part given by Eqs. (4b). At the peak of the phonon drag near $4 \mathrm{~K}$, the ratio of former to the latter is about 60 . If
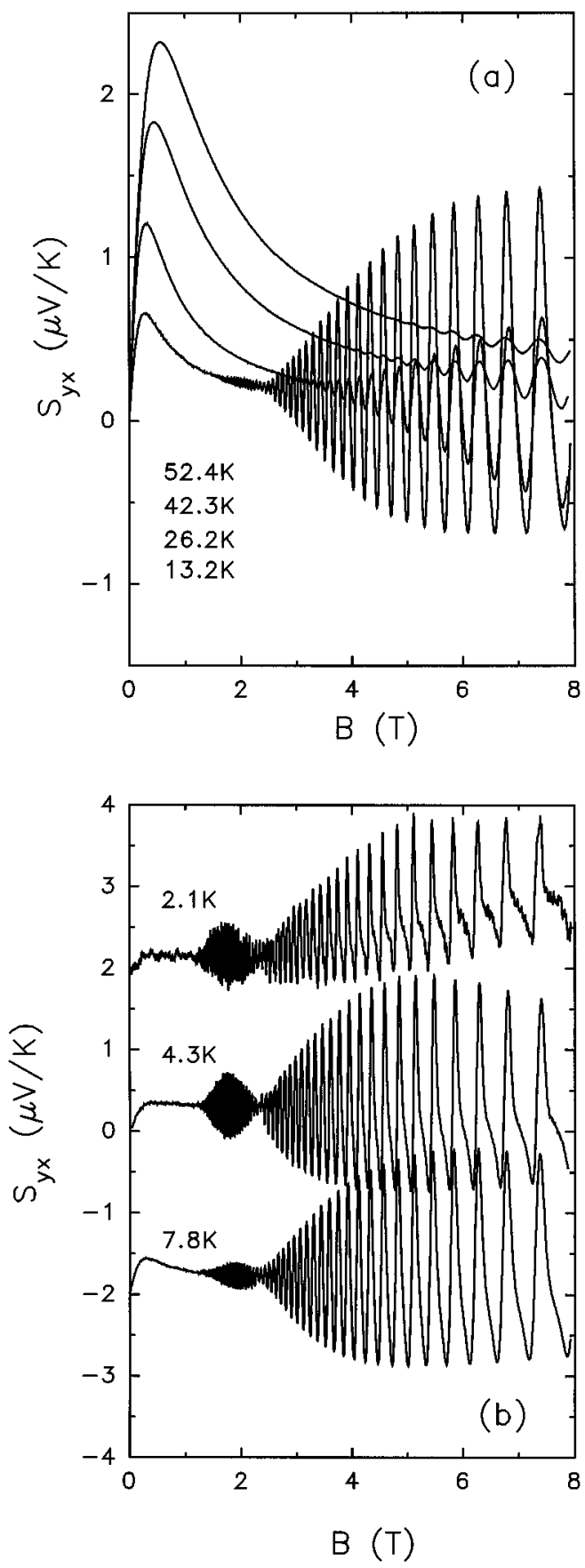

FIG. 5. Examples of the Nernst-Ettingshausen coefficient $S_{y x}$ as a function of magnetic field. Data in panel (a) are at higher temperatures, and those in panel (b) at lower temperatures. In panel (b) the curves at 2.1 and $7.8 \mathrm{~K}$ have been offset by $2 \mu \mathrm{V} / \mathrm{K}$.

perfect cancellation of the two phonon drag terms did not occur, the residual would be easily recognizable since it would not be linear in $T$, as was found with a $2 \mathrm{DEG} .{ }^{10}$ There may indeed be a tiny peak near $4 \mathrm{~K}$ in Fig. 6, possibly reflecting the peak in Fig. 4, though the noise is similar in magnitude at these low temperatures. In any event the results imply that the two terms are matched to no worse than $\sim 1 \%$. With $\varepsilon_{F}=0.155 \mathrm{eV}$ in Eq. (4b), we find $p=-0.57$.

We have used Eq. (6b) and the measured $\widetilde{\rho}_{x x}$ (the contribution from $\tilde{\rho}_{y x}$ is completely negligible) to calculate the oscillatory contribution $\widetilde{S}_{y x}$. The phase has been shifted by 


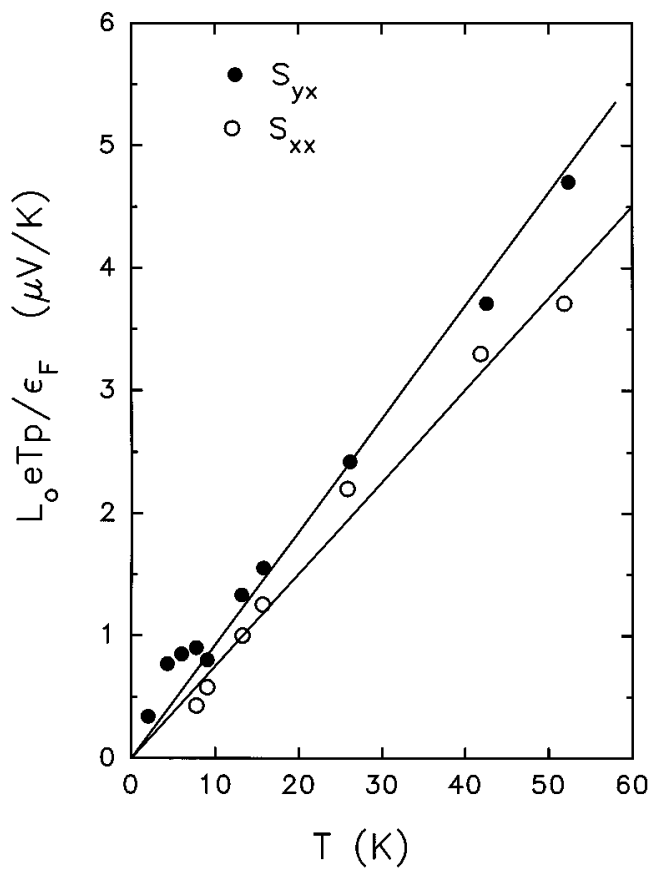

FIG. 6. The quantity $L_{o} e T p / \varepsilon_{F}$ obtained from fits to the nonoscillatory parts of $S_{y x}$ and $S_{x x}$, respectively, as a function of temperature.

$\pi / 2$, and we use the same mobility $\mu$ as required for the smooth background. At low temperatures the harmonics in $\tilde{\rho}_{x x}$ become very large, and must be taken into account to obtain the observed wave form for $\widetilde{S}_{y x}^{d}$ Thus we have extracted the fundamental and the first two harmonics using Fourier analysis, evaluated these contributions separately in the analysis, and finally added them and the nonoscillatory parts to give the results shown in Fig. 7. Notice there are no free parameters in this calculation. The correspondence between experiment and calculation is excellent at all temperatures and fields. We particularly draw attention to the triangular wave form at low temperatures which is well reproduced by the harmonic contributions. The sharp peaks of the calculated data superpose essentially perfectly onto the experimental data, both in magnitude and position.

The phases of $\widetilde{\rho}_{x x}$ and $\widetilde{S}_{y x}$ have also been compared directly from the experimental data using a method described previously. ${ }^{20}$ In the range with the highest experimental accuracy $(B=5-8 \mathrm{~T}$ and $T>15 \mathrm{~K})$, all the $\widetilde{\rho}_{x x}$ data are found to have the same phase to an accuracy of $\leqslant \pm \pi / 10$, and the same is true for $\widetilde{S}_{y x}$. The two averages differ by almost exactly $\pi / 2$, as predicted.

Figure 8 shows typical data for $S_{x x}$ as a function of $B$ at various temperatures. Above $\sim 9 \mathrm{~K}$ the nonoscillatory part shows a rapid rise followed by saturation. This is the effect of the second term containing $p$ in Eq. (4a) for $\bar{S}_{x x}^{d}$, and, because $p$ is negative, $\bar{S}_{x x}^{d}$ increases with field. At lower temperatures this rise becomes swamped by the rapidly increasing phonon drag contribution, which, according to Eq. (8a), is independent of field. There is also a decrease with $B$ at lower temperatures which is clearly related to the field dependence of $\lambda_{x x}$ described in Sec. IV A. A decrease in $\lambda_{x x}$ implies more field-dependent phonon scattering (other
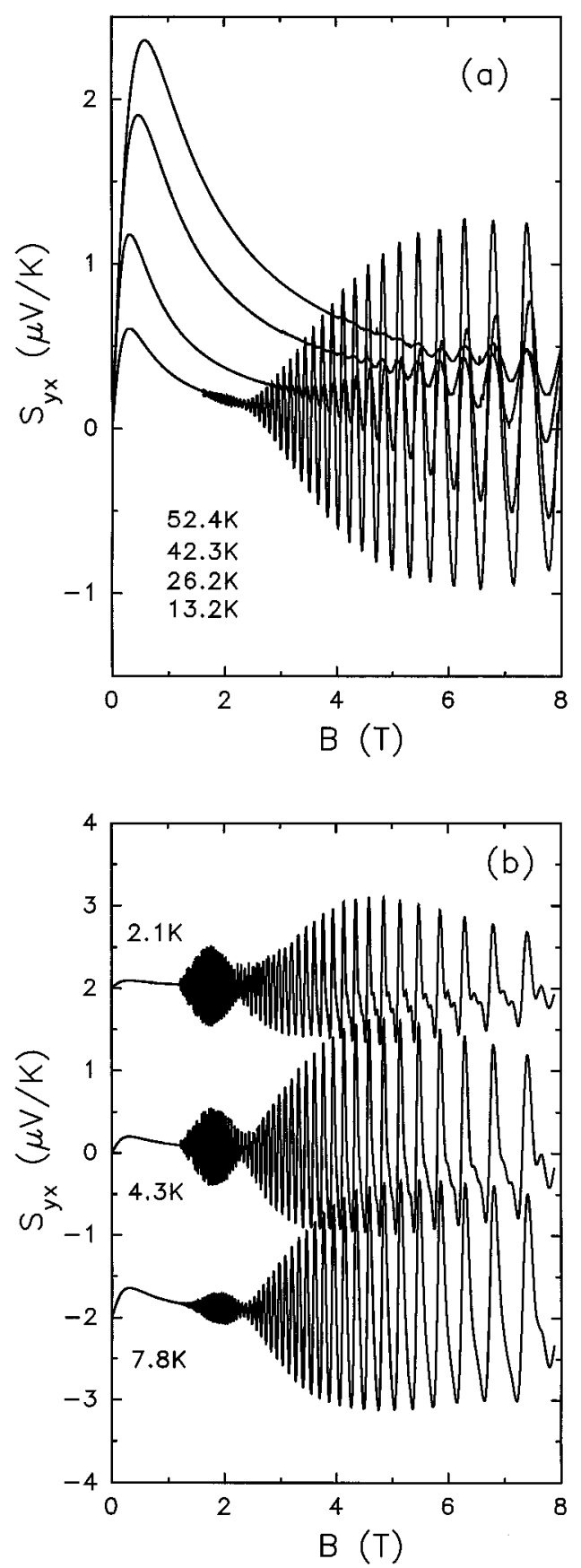

FIG. 7. Calculated $S_{y x}$ for the same temperatures as the experimental data in Figs. 5(a) and 5(b). In panel (b) the curves at 2.1 and $7.8 \mathrm{~K}$ have been offset by $2 \mu \mathrm{V} / \mathrm{K}$.

than $p$-e scattering), and consequently a decrease in phonon drag, but the origin of this feature is unknown.

Above $\sim 10 \mathrm{~K}$ the field dependence of the nonoscillatory background has been fitted to Eq. (4a), with an additive constant to take phonon drag into account. The fits give $\mu$ consistent with those from $\bar{S}_{y x}$, and $L_{o} e T p / \varepsilon_{F}$ is shown in Fig. 6 . We find $p=-0.48$ compared to the value of -0.57 from $\bar{S}_{y x}$. The discrepancy is rather small, and might simply reflect errors in sample dimensions, but it can also arise from the fact that the electronic thermal conductivity is not negligible above about $20 \mathrm{~K}$. This means $\nabla T_{y} \neq 0$ which, in turn, implies that the thermopower and the NE coefficient are no 

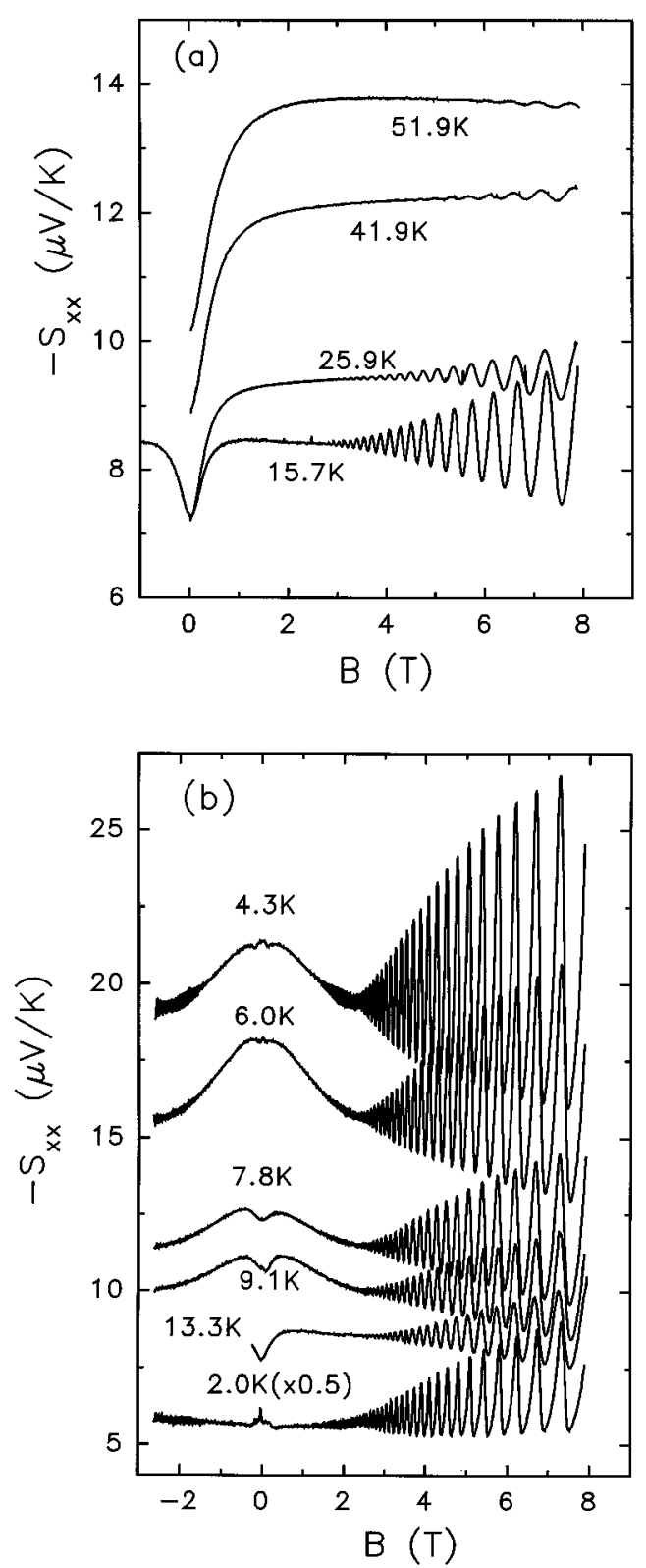

FIG. 8. Examples of the thermopower $S_{x x}$ as a function of field at various temperatures. Notice the scale change for the data at 2.0 $\mathrm{K}$.

longer precisely equal to $S_{x x}$ and $S_{y x}$, respectively. For example, one finds that the NE coefficient is approximately given by $S_{y x}-S_{x x}\left(\lambda_{y x}^{e} / \lambda_{x x}\right)$. At about $50 \mathrm{~K}$ this will appear to increase the magnitude of the NE coefficient by perhaps $20 \%$, which leads to a value of $p$ too high by the same factor. Although a similar relation holds for the thermopower, in these experiments this correction is negligible. The results for $p$ are both consistent with the value of -0.5 expected from a constant electronic mean free path.

We have used $p=-0.50$ and Eq. (4a) to calculate the diffusion component of the zero-field thermopower $S_{o}$ as a function of $T$, and this is the straight line shown in Fig. 4. We also note that, at least above about $10 \mathrm{~K}$, the consistency of the analysis in using $\bar{S}_{x x}^{d}$ to describe the field dependence of the measured thermopower means that the nonoscillatory part of the phonon drag is independent of field, in agreement with Eq. (8a).

The oscillatory part $\widetilde{S}_{x x}$ is accurately $\pi$ out of phase with $\tilde{\rho}_{x x}\left(S_{x x}\right.$ is a negative quantity) over the whole temperature range, which immediately shows that Eq. (6a) cannot represent these data. The calculated amplitude is also too small at all temperatures. At $8 \mathrm{~T}$ and $4 \mathrm{~K}$ it predicts $0.07 \mu \mathrm{V} / \mathrm{K}$ compared with the observed $13 \mu \mathrm{V} / \mathrm{K}$. The agreement improves at high temperature, but is never good. At $50 \mathrm{~K}$ the measured amplitude at $8 \mathrm{~T}$ is $\sim 0.08 \mu \mathrm{V} / \mathrm{K}$, compared with the calculated value of $0.03 \mu \mathrm{V} / \mathrm{K}$.

The entropy result of Eq. (7) has the correct phase, but is also unsuccessful with the amplitude. For $T<8 \mathrm{~K}$ and $B=8 \mathrm{~T}, X<1$, so that $\widetilde{S}_{x x}^{d} / \bar{S}_{x x}^{d}(\infty)=\widetilde{\nu} / \bar{\nu}$ is a good approximation. Using $\widetilde{\nu} / \bar{\nu} \sim 0.4$, Eq. (7) predicts $\widetilde{S}_{x x}^{d} \sim 0.4 \bar{S}_{x x}^{d}(\infty)$. Because $\bar{S}_{x x}^{d}(\infty) \sim(4 / 3) S_{o}^{d}$, and Fig. 4 shows that $S_{o}^{d}$ very small compared to $S_{o}^{g}$ then these oscillations are also negligible. At high temperatures the agreement improves as with Eq. (6a). At $50 \mathrm{~K}$ and $8 \mathrm{~T}$ the calculated amplitude is $\sim 0.05 \mu \mathrm{V} / \mathrm{K}$.

The poor agreement with either of the above diffusion results, especially at low temperatures, leads to the inevitable conclusion that the oscillations have their origin in phonon drag according to Eq. (8a). In other words, they reflect the changes induced in phonon-electron scattering due to magnetic quantization of the electrons. This identification does not appear to have been made before for degenerate semiconductors, but it is an obvious extension of the 2D case, which is now well known. ${ }^{3}$ At low temperatures the observed $\widetilde{S}_{x x} / \bar{S}_{x x}$ is about $50 \%$, in accordance with $\gamma \sim 1$. When $k_{B} T \ll \hbar \omega_{c}$, we would expect the oscillation amplitude to scale with $\bar{S}_{x x}$. At $8 \mathrm{~T}$ this should be so for $T \sim 2-10 \mathrm{~K}$. Figure 8 does indeed show the expected scaling which is especially convincing given the strong variation of $\bar{S}_{x x}$ in this temperature range. For comparison, we note that both Eqs. (7) and (6a) predict a scaling as $T$ under these conditions. Of course, at higher temperatures, when $k_{B} T \ll \hbar \omega_{c}$ no longer holds, all contributions to the oscillations are thermally damped. For elastic scattering the damping term is $D^{\prime}(X)$ in Eqs. (6a) and (7), but the form of the damping term is not known for $\widetilde{S_{x x}^{g}}$ and so we cannot estimate the relative contributions from diffusion and phonon drag to the hightemperature oscillations.

Finally, we briefly return to the oscillations found in $\lambda_{x x}^{g}$ mentioned in Sec. IV A. An estimate of $\widetilde{\lambda}_{x x}^{g}$ can be made as follows. Let the relaxation time for $p$-e scattering be $\tau_{e}$, and that for phonon scattering by all other mechanisms $\tau_{a}$. Then $\lambda_{x x}^{g} / \lambda_{o}^{g}=\left(1 / \tau_{e o}+1 / \tau_{a o}\right) /\left(1 / \tau_{e}+1 / \tau_{a}\right)$, where the extra subscript $o$ specifies zero field. With some re-arrangement this gives $\lambda_{x x}^{g} / \lambda_{o}^{g}=\left(\tau_{a} / \tau_{a o}\right)\left(1+\eta_{o}\right) /\left[1+\eta_{o}\left(\tau_{e o} / \tau_{a o}\right)\left(\tau_{a} / \tau_{e}\right)\right]$, where $\eta_{o}=\tau_{a o} / \tau_{e o}$ is the ratio of scattering of phonons by electrons to scattering by all other mechanisms at zero field. Elementary models ${ }^{21}$ of phonon drag in $2 \mathrm{D}$ or 3D metals give the relation $S_{o}^{g}=$ const $\times C^{g}\left(\tau_{a o} / \tau_{e o}\right) /\left(1+\eta_{o}\right)$ $\sim C^{g}\left(\tau_{a o} / \tau_{e o}\right)$ where $C^{g}$ is the lattice specific heat. Using this together with the similar relation $S_{x x}^{g} \sim C^{g}\left(\tau_{a} / \tau_{e}\right)$, we finally find 


$$
\frac{\lambda_{x x}^{g}}{\lambda_{o}^{g}} \simeq \frac{\tau_{a}}{\tau_{a o}} \frac{\left(1+\eta_{o}\right)}{\left[1+\eta_{o}\left(S_{x x}^{g} / S_{o}^{g}\right)\right]}
$$

This should be appropriate when all the phonons participate in both $\lambda^{g}$ and $S^{g}$, i.e., over the range $1-6 \mathrm{~K}$ approximately. The nonoscillatory variation $\bar{\lambda}_{x x}^{g}$ is caused by $\tau_{a} / \tau_{a o}$, and the oscillatory part $\widetilde{\lambda}_{x x}^{g}$ arises through the term $S_{x x}^{g}$. Using the experimental data for $S_{x x}$ (which is essentially $S_{x x}^{g}$ ) as a function of $B$, we have calculated $\lambda_{x x}^{g} / \lambda_{o}^{g}$ at 2 and $6 \mathrm{~K}$ using values of $\tau_{a} / \tau_{a o}$ required to approximately match the background at high fields and $\eta_{o}=0.09$, and the results are in good agreement with experiment as shown in Fig. 2.

The required fraction of scattering by electrons is in keeping with our theoretical estimates in Sec. IV A. Thus in Fig. 2 the fitted curve for $\lambda_{o}^{g}$ is given both with and without $p$ $e$ scattering. The calculated ratio $\eta_{o}$ varies from $\sim 0.15$ at 1 $\mathrm{K}$ to 0.01 at $10 \mathrm{~K}$. However, by about $9 \mathrm{~K}$ the observed oscillations in $\lambda_{x x}^{g}$ show that electronic scattering is larger than our calculation predicts, which probably implies that the calculation of $p-e$ scattering is in error at higher temperatures.

\section{CONCLUSIONS}

Our model calculation of the thermopower tensor $S$ for a degenerate semiconductor predicts that both components $S_{x x}$ and $S_{y x}$ have diffusion contributions, but that only the former will show phonon drag effects. Experimentally this is indeed found to be so for both the oscillatory and nonoscillatory parts. This remarkable difference between the components is clearly a very useful result which has not been previously recognized. One would expect 2DEG's to act in the same way, but a phonon drag component of $S_{y x}$ has definitely been seen ${ }^{10}$ in that case, though it is small. The reason for this discrepancy is not known, and deserves close attention. These two cases are particularly simple experimentally because the absence of a transverse temperature gradient means that the NE coefficient gives $S_{y x}$ directly, and similarly the thermopower gives $S_{x x}$.

The diffusion model we have used to describe both the oscillatory and nonoscillatory components of $S_{y x}$ has been accurately confirmed. In particular, the oscillations in $S_{y x}$ show excellent agreement with calculated amplitudes, and also the expected phase shift of $\pi / 2$ compared to resistance oscillations. In contrast, the oscillations in $S_{x x}$ are found to be due almost entirely to phonon drag, as is also true in 2DEG's. We have given a semiquantitative argument for the amplitude of the phonon drag oscillations at low temperatures and high magnetic fields, but a full quantitative calculation is lacking. An analysis of the oscillations in the lattice thermal conductivity at low temperatures supports the identification of the oscillations in $S_{x x}$ with phonon drag.

Previous experimental data on oscillations in the thermopower of degenerate semiconductors, particularly on $\mathrm{HgSe}^{8}$ have been analyzed in terms of entropy oscillations, but we have shown these cannot be the cause, at least at low temperatures. It is unfortunate that we cannot see recognizable diffusion oscillations in $S_{x x}$, because this means we are unable to distinguish which, if either, of the models for these oscillations is correct for this coefficient.
In bulk metals, diffusion oscillations are dominant in both the thermopower and NE coefficient, and conform to Eq. (5), but most investigations have involved magnetic breakdown, so the situation is perhaps not completely clear cut. Phonon drag oscillation have not been seen, which agrees with our contention that these are at the same relative level as the oscillations in the electronic density of states; these latter are typically at a level of $1 \%$ or less as a result of the high electronic density.

\section{ACKNOWLEDGMENTS}

We are very grateful to L.W.M. Scheurs for technical support in sample preparation and to Dr. Eugene Zaremba for illuminating discussions concerning phonon drag thermopower. This work was supported, in part, by a grant from the Natural Sciences and Engineering Research Council of Canada, and is a part of a research program of the Stichting voor Fundamenteel Onderzoek der Materie (FOM) financially supported by NWO (The Netherlands).

\section{APPENDIX A: ENTROPY OSCILLATIONS AND THERMOPOWER}

Equation (7) is from the high-field limit of Eq. (2a), i.e., $S_{x x}^{d}=\rho_{y x} \epsilon_{x y}^{d}$ with $\rho_{y x}$ taken to be $B / n e$ and nonoscillatory. It is well known that, as $\beta \rightarrow \infty, \bar{\epsilon}_{x y}^{d}$ is a direct measure of the nonoscillatory density of states at the Fermi level by virtue of the Mott relation applied to $\bar{\sigma}_{x y}=n e / B$. It therefore seems reasonable that the oscillatory density of states would appear in $\widetilde{\epsilon}_{x y}$, and this is what Eq. (7) claims for the oscillatory part $\widetilde{S}_{x x}^{d}$. However, other arguments suggest otherwise.

All calculations of $\sigma_{x y}$ taking magnetic quantization into account are consistent with this coefficient having no oscillations as $\beta \rightarrow \infty$. Guseva and Zyryanov ${ }^{22}$ and Horton ${ }^{23}$ find that [ignoring the thermal damping term $D(X)$ ]

$$
\begin{aligned}
\sigma_{x y}= & \frac{n e}{B}\left\{1+\frac{7}{2 \beta^{2}}\right. \\
& \left.\times\left[\left(\frac{\hbar \omega_{c}}{2 \varepsilon_{F}}\right)^{1 / 2} \sum_{r} \frac{(-1)^{r}}{\sqrt{r}} \cos \left(\frac{2 \pi \varepsilon_{F} r}{\hbar \omega_{c}}-\frac{\pi}{4}\right)\right]\right\} .
\end{aligned}
$$

The oscillatory corrections are a result of residual scattering effects, whereby the electron-scattering probability reflects the oscillations in the density of states, and go to zero as $\beta \rightarrow \infty$. The absence of oscillations as a function of $B$ in the zero-scattering limit also implies there are no oscillations in the energy dependence of this coefficient. This can be seen directly from Eq. (10) where one can fix either $\varepsilon_{F}$ or $B$ and allow the other to vary. However, it is the zero-scattering limit that Obraztsov uses to calculate $\widetilde{\epsilon}_{x y}$. The combination of these two results implies that $\widetilde{\epsilon}_{x y}^{d}$ and $\widetilde{\sigma}_{x y}$ are not related in this limit and cannot, for example, satisfy the Mott result which would be expected to hold when $k_{B} T \ll \hbar \omega_{c}$.

In contrast Eq. (5) is essentially the Mott result extended to the case of oscillations in the density of states with arbi- 
trary $k_{B} T / \hbar \omega_{c}$, and cancels to the usual Mott relation given above in the high-field limit $k_{B} T \ll \hbar \omega_{c}$. In the zeroscattering limit, Eq. (10) predicts $\widetilde{\sigma}_{x y}=0$, and this means $\widetilde{\epsilon}_{x y}=0$ from Eq. (5). Lest it be assumed that this result has no basis other than Young's ${ }^{5}$ semiclassical arguments, they are supported by a quantum-mechanical density matrix calculation by Horton ${ }^{23}$ for free electrons. Horton gives results for $\widetilde{\sigma}_{y x}[\mathrm{Eq} .(10)]$ and $\widetilde{\epsilon}_{x y}$ which are consistent with Eq. (5). Thus the two results Eqs. (5) and (7) appear to be inconsistent. A resolution of this problem is clearly called for.
${ }^{1}$ P. S. Zyrayanov and G. I. Guseva, Usp. Fiz. Nouk 95, 565 (1968) [Sov. Phys. Usp. 11, 538 (1969)].

${ }^{2}$ S. M. Puri and T. H. Geballe, Physics of III-V Compounds, edited by R. K. Willardson and A. C. Beer, Semiconductors and Semimetals Vol. 1 (Academic, New York, 1966), p. 203.

${ }^{3}$ B. L. Gallagher and P. N. Butcher, in Handbook on Semiconductors, edited by P. T. Landsberg (Elsevier, Amsterdam, 1992), Vol. 1, p. 817.

${ }^{4}$ R. Fletcher, P. T. Coleridge, and Y. Feng, Phys. Rev. B 52, 2823 (1995).

${ }^{5}$ R. C. Young, J. Phys. F 3, 721 (1973).

${ }^{6}$ R. Fletcher, J. Low Temp. Phys., 43, 363 (1981).

${ }^{7}$ R. Fletcher, Phys. Rev. B 28, 1721 (1983); 28, 6670 (1983).

${ }^{8}$ B. Schroder and G. Landwehr, Solid State Commun. 22, 589 (1977).

${ }^{9}$ J. M. Ziman, Electron and Phonons (Clarendon, Oxford, 1963), p. 500.

${ }^{10}$ R. Fletcher, J. J. Harris, C. T. Foxon, M. Tsaousidou, and P. N. Butcher, Phys. Rev. B 50, 14991 (1994).

${ }^{11}$ I. G. Kuleev, A. T. Lonchakov, I. I. Lyapilin, and M. Tsidil'kovskii, Zh. Eksp. Teor. Fiz. 103, 1447 (1993) [Sov. Phys. JETP 76, 707 (1993)].

${ }^{12}$ L. M. Roth and P. N. Argyres, in Physics of III-V Compounds (Ref. 2), p. 159.
${ }^{13}$ Yu. N. Obraztsov, Fiz. Tverd Tela (Leningrad) 7, 573 (1965) [Sov. Phys. Solid State 7, 455 (1965)].

${ }^{14}$ E. Zaremba (private communication); (unpublished).

${ }^{15}$ X. Zianni, P. N. Butcher, M. J. Kearney, Phys. Rev. B 49, 7520 (1994).

${ }^{16}$ M. von Ortenberg, Semicond. Sci. Technol. 8, S16 (1993).

${ }^{17}$ M. Vaziri and R. Reifenberger, Phys. Rev. B 32, 3921 (1985); 33, 5585 (1986).

${ }^{18}$ C. W Whitsett, D. A. Nelson, J. G. Broerman, and E. C. Paxhia, Phys. Rev. B 7, 4625 (1973).

${ }^{19}$ M. N. Wybourne, C. G. Edison, and M. J. Kelly, J. Phys. C 17, L607 (1984).

${ }^{20}$ R. Fletcher, J. J. Harris, and C. T. Foxon, J. Phys. Condens. Matter 3, 3479 (1991).

${ }^{21}$ For 2 D, see M. J. Smith and P. N. Butcher, J. Phys. Condens. Matter 2, 2375 (1990). For 3D, see R. D. Bernard, Thermoelectricity in Metals and Alloys (Taylor and Francis, London, 1972), p. 113.

${ }^{22}$ G. I. Guseva and P. S. Zyryanov, Phys. Status Solidi 25, 775 (1968).

${ }^{23}$ Philip B. Horton, Ph.D. thesis, Louisiana State University, 1964 (unpublished). Many of Horton's results are given in J. R. Long, C. G. Grenier, and J. M. Reynolds, Phys. Rev. 140, A187 (1965). 